\title{
Identification of stable genes in the corpus luteum of lactating Holstein cows in pregnancy and luteolysis: Implications for selection of reverse-transcription quantitative PCR reference genes
}

\author{
M. A. Mezera, ${ }^{1,2} \odot$ W. Li, ${ }^{3 *}$ A. J. Edwards, ${ }^{3}$ D. J. Koch, ${ }^{3}$ A. D. Beard,,${ }^{1,2} \odot$ and M. C. Wiltbank ${ }^{1} \odot$ \\ ${ }^{1}$ Department of Dairy Science, University of Wisconsin, Madison 53706 \\ ${ }^{2}$ Endocrinology and Reproductive Physiology Program, University of Wisconsin, Madison 53706 \\ ${ }^{3}$ USDA Dairy Forage Research Center, Madison, WI 53706
}

\begin{abstract}
In lactating dairy cattle, the corpus luteum (CL) is a dynamic endocrine tissue vital for pregnancy maintenance, fertility, and cyclicity. Understanding processes underlying luteal physiology is therefore necessary to increase reproductive efficiency in cattle. A common technique for investigating luteal physiology is reversetranscription quantitative PCR (RT-qPCR), a valuable tool for quantifying gene expression. However, reference-gene-based RT-qPCR quantification methods require utilization of stably expressed genes to accurately assess mRNA expression. Historically, selection of reference genes in cattle has relied on subjective selection of a small pool of reference genes, many of which may have significant expression variation among different tissues or physiologic states. This is particularly concerning in dynamic tissues such as the CL, with its capacity for rapid physiologic changes during luteolysis, and likely in the less characterized period of CL maintenance during pregnancy. Thus, there is a clear need to identify reference genes well suited for the bovine CL over a wide range of physiological states. Whole-transcriptome RNA sequencing stands as an effective method to identify new reference genes by enabling the assessment of the expression profile of the entire pool of mRNA transcripts. We report the identification of 13 novel putative reference genes using RNA sequencing in the bovine CL throughout early pregnancy and luteolysis: RPL4, UQCRFS1, COX 4I1, RPS4X, SSR3, CST3, ZNF266, CDC42, CD63, HIF1A, $Y W H A E, E I F 3 E$, and PPIB. Independent RT-qPCR analyses were conducted confirming expression stability in another set of CL tissues from pregnancy and regression, with analyses performed for 3 groups of
\end{abstract}

Received August 30, 2019.

Accepted January 10, 2020.

*Corresponding author: wenli.li@usda.gov samples: (1) all samples, (2) samples from pregnancy alone, and (3) samples throughout the process of CL regression. Seven genes were found to be more stable in all states than 2 traditional reference genes $(A C T B$ and GAPDH): RPS4X, COX 4I1, PPIB, SSR3, RPL4, $Y W H A E$, and $C D C 42$. When CL tissues from pregnant animals alone were analyzed, CST3, HIF1A, and CD63 were also identified as more stable than $A C T B$ and $G A P D H$. Identification of these new reference genes will aid in accurate normalization of RT-qPCR results, contributing to proper interpretation of gene expression relevant to luteal physiology. Furthermore, our analysis sheds light on the effects of luteolysis and pregnancy on the stability of gene expression in the bovine CL.

Key words: corpus luteum, dairy cow, reference gene, reverse-transcription quantitative PCR

\section{INTRODUCTION}

Vital for reproductive efficiency, the corpus luteum (CL) is a highly dynamic endocrine gland, undergoing alteration of over 900 genes in regression (Mondal et al., 2011; Ochoa et al., 2018), and 265 genes by the second month of pregnancy (Sakumoto et al., 2015). Although these dynamics are critically important to luteal physiology, this introduces challenges in interpreting reversetranscription quantitative PCR (RT-qPCR) data because this method is dependent on normalization (Huggett et al., 2005). Although newer techniques such as RNA sequencing (RNA-seq) reduce this challenge (Wang et al., 2009), RT-qPCR remains popular due to its low cost and accessibility. Thus, despite the suboptimal nature of RT-qPCR, improvements to RT-qPCR data analyses remain relevant.

Currently, a commonly adopted normalization procedure for RT-qPCR uses a few de facto reference genes, including $A C T B$ and GAPDH, despite increasing evidence indicating instability in some conditions (Suzuki et al., 2000; Glare et al., 2002; Steele et al., 2002; Bas et al., 2004). This can alter interpretation of results 
(Huggett et al., 2005) and confound the understanding of physiology (Bas et al., 2004). To address these shortcomings, efforts have been made to find genes more stable than GAPDH and $A C T B$ in bovine CL (Pfaffl et al., 2004; Rekawiecki et al., 2012; Schoen et al., 2015). However, genes for analysis were selected without knowledge of the whole transcriptome, limiting the number of genes considered for normalization. Thus, a large pool of potentially stably expressed mRNA transcripts have not been previously considered in the CL, nor has there been sufficient consideration of physiologic state on gene stability (e.g., regression or pregnancy).

With its high sensitivity and repeatability (Chen et al., 2017), RNA-seq provides an effective tool to rapidly consider all transcripts for suitability as reference genes (de Jonge et al., 2007). In this study, a series of biopsies from regression and pregnancy were sequenced to identify stable genes, and stability was confirmed in an independent set of samples via RT-qPCR. Performance was compared with the 2 most common reference genes in bovine CL literature: GAPDH and $A C T B$. This led to the discovery of new reference genes in the following conditions: in cycling animals before, during, and after regression; in the first 2 mo of pregnancy; and when samples from these conditions were analyzed together. Although no single set of reference genes is optimal for use in all experiments without validation, the present report provides an evidence-based starting point for selection of reference genes in future studies of luteal physiology and identifies differences in gene stability in regression and pregnancy.

\section{MATERIALS AND METHODS}

\section{Animals, Experimental Design, Sample Collection, and Animal Classification}

All animal procedures were approved by the University of Wisconsin-Madison Institutional Animal Care and Use Committee. Multiparous Holstein cows were housed in a tiestall barn, milked twice per day, and fed a standard herd diet. As previously described (Mezera et al., 2019), 32 cows were synchronized, with half bred and half uninseminated. Blood was collected from the jugular vein on d 18 to 21 to analyze circulating progesterone $(\mathbf{P} 4)$ and $\mathrm{PGF}_{2 \alpha}$ metabolite (PGFM) to determine regression status, while biopsies were collected daily from d 18 to 21 with a transvaginal guided ultrasound probe, as described and validated by Tsai et al. (2001). The CL biopsies were stored in $100 \mu \mathrm{L}$ of RNALater (R0901, Sigma-Aldrich, Darmstadt, Germany) at $-80^{\circ} \mathrm{C}$ until analyzed. In another group of pregnant animals, CL biopsies were collected at $55 \pm 3$ d of pregnancy.

Upon evaluation of P4 and PGFM, a total of 32 samples from the following categories were sequenced: (1) d 20 of pregnancy $(\mathrm{n}=5)$; (2) d 50 to 64 of pregnancy $(\mathrm{n}=4)$; (3) late luteal phase (d 18-20 of cycle, nonbred), no evidence of CL regression ( $\mathrm{n}=10)$; (4) early regression: exposed to $\mathrm{PGF}_{2 \alpha}$ but no significant decline in $\mathrm{P} 4(\mathrm{n}=8)$; and (5) late regression $(\mathrm{P} 4<1$ $\mathrm{ng} / \mathrm{mL} ; \mathrm{n}=5)$.

\section{RNA Isolation and RNA-seq}

Samples were depleted of RNAlater and RNA isolated using the RNeasy Micro Kit with DNase treatment (74034, Qiagen, Hilden, Germany). The RNA quality was assessed using the Agilent RNA 6000 Pico Chip (5067-1513, Agilent) on the Agilent 2100 Bioanalyzer. The RNA quantity was assessed with the Qbit RNA HS Assy Kit (Q32852, Thermo Fisher Scientific, Waltham, MA).

Library preparation was completed using the Illumina TruSeq Stranded Total RNA (LT) kit (RS-1222302, Illumina, San Diego, CA) with 400 ng of RNA as starting material and addition of $1 \mu \mathrm{L}$ of $1: 10$ diluted ERCC (4456740, Thermo Fisher Scientific) substituted for $1 \mu \mathrm{L}$ of sample before addition of Elute, Prime, Fragment High Mix. Fragment distribution of the library was assessed with the Agilent DNA 1000 Chip (5067-1505, Agilent). Library concentration was assessed using Kapa library quantification kit (KK4824, Kapa Biosystems, Wilmington, MA). Pooled libraries were sequenced with the NextSeq 500/550 High Output Kit v2 (FC-404-2002, Illumina).

\section{RNA-seq Raw Reads Mapping and Data Analysis}

Quality of raw reads was checked using FastQC (https: / / www.bioinformatics.babraham.ac.uk/ projects/fastqc/). For sequence alignment, NCBI UMD3.1 (https://www.ncbi.nlm.nih.gov/assembly/ GCF_000003055.6/), Bos taurus reference genome was used. Raw reads from all whole transcriptome RNA-seq libraries were aligned using a 2-step approach. First, Tophat2 (Kim et al., 2013) was used with the following settings: "-r 70-mate-std-dec 90" for paired-end reads. Second, unmapped reads from step 1 were realigned with Bowtie2 (Langmead and Salzberg, 2012) using the "-very-sensitive-local" method. The genome annotation file (NCBI, UMD3.1) downloaded from the Tophat website (http://ccb.jhu.edu/software/tophat/ igenomes.shtml) was used as reference. Genes shorter than $150 \mathrm{bp}$ were excluded from the GTF file. Raw 
reads shorter than 35 bp were excluded from the alignment process. Combined (Tophat + bowtie2) sequence alignment generated by the 2-step alignment approach served as input file for HTSeq. Raw read counts for each gene were obtained using HTSeq (v0.6; Anders et al., 2015).

Normalizations of mRNA were performed using 2 methods: (1) expression of mRNA in each sample were normalized to fragments per kilobase of cDNA per million fragments mapped (FPKM) using cufflinks (Trapnell et al., 2012); and (2) the raw read counts generated by HTSeq were normalized using the read counts of spike-in ERCC by Bioconductor package RUV (Risso et al., 2014). Normalized read count values were used to assess gene expression profiles for each sample.

\section{Selection of Stably Expressed Genes Using RNA-seq Data}

To identify stably expressed genes, 2 normalized gene read-counts were used: FPKM values and ERCCcorrected read-counts. First, using the FPKM values, the following procedure was performed: (1) all samples were labeled by physiological state; (2) for each gene, ANOVA analysis was performed using the FPKM values to compare among the samples grouped by physiologic state; and (3) genes with $P$-value $\geq 0.1$ and standard deviation less than $20 \%$ of the mean (using each sample individually without regard to physiologic state) were kept for further analysis. Finally, to account for any potential variation introduced by sequencing batches, all the kept genes (after the previous 3 steps) were further analyzed for their differences in expression using DEseq2 with raw read counts as the input value. Because DEseq2 was developed to compare 2 groups, the samples were randomly assigned to 2 equal groups regardless of original category for this calculation. Genes with $P$-value $<0.1$ were not considered to be stably expressed. Second, using the ERCC-normalized read-counts for each gene, a list of potential stable reference genes was generated by the same process, except for step 3, where genes were accepted with a standard deviation less than $30 \%$ of the mean. Code used for data analysis can be found at https:/github.com/ WLpython19/luteal-Reference-gene.

ToppGene (Chen et al., 2009) was used to identify pathways enriched in the stably expressed genes. Using ANOVA analysis with FPKM values, stably expressed genes were further analyzed for enrichment of biological pathways using the Biosystems:REACTOME categories (Fabregat et al., 2018). Pathways were considered significant when Bonferroni-corrected $P$-value was $<0.05$.

\section{Review of Previously Used Reference Genes from the Literature}

Relevant published papers were located in the Web of Science core collection using the search terms "corpus luteum" (title), and "bovine" (topic) published in the last 5 yr (search conducted November 18, 2018). Reference genes of every paper utilizing RT-qPCR were recorded, and the 2 most common genes (GAPDH and $A C T B$, Supplemental Table S1, https://doi.org/10 .3168/jds.2019-17526) selected to provide a comparison for validation of new reference genes.

Further consideration of novel genes was conducted by noting fold changes and Q-values from DEseq2 analysis of the RNA-seq data set comparing expression of mRNA in the most dynamic comparison: post regression $(\mathrm{P} 4<1 \mathrm{ng} / \mathrm{mL})$ versus late luteal phase CL.

\section{RT-qPCR Analysis of Proposed Reference Genes}

Twenty-three independent CL samples were used for RT-qPCR validation, which consisted of samples from animals in the following groups: pregnant $\mathrm{d} 18, \mathrm{n}=$ 4 ; d $52.3 \pm 3.4$ of pregnancy, $\mathrm{n}=4$; late luteal phase (cycling d 18, no regression in the following $24 \mathrm{~h}$ ), $\mathrm{n}=$ 5 ; late regression $(\mathrm{P} 4<1 \mathrm{ng} / \mathrm{mL}), \mathrm{n}=5$; and early regression (PGFM exposure, no drop in $\mathrm{P} 4), \mathrm{n}=5$.

The RNA was extracted with the RNeasy Micro Kit (74004, Qiagen). The cDNA was generated using the High Capacity cDNA Reverse Transcription Kit (4368814, Applied Biosystems, Waltham, MA). Gene expression was measured using the CFX Connect RealTime System (Bio-Rad, Hercules, CA) using $5 \mu \mathrm{L}$ of SsoFast EvaGreen Supermix (172-5203, Bio-Rad), 2 $\mu \mathrm{L}$ of cDNA, $500 \mathrm{n} M$ each of forward and reverse primers [Supplemental Table S1, https://doi.org/10.3168/ jds.2019-17526, designed with Primer-BLAST (Ye et al., 2012)], and water to bring the reaction to $10 \mu \mathrm{L}$. The thermal cycling conditions were $95^{\circ} \mathrm{C}$ for $3 \mathrm{~min}$, then 40 cycles of $95^{\circ} \mathrm{C}$ for $10 \mathrm{~s}$ and $60^{\circ} \mathrm{C}$ for $30 \mathrm{~s}$.

\section{Analysis of Proposed Reference Genes}

Results of PCR were analyzed using 3 algorithms: NormFinder (Andersen et al., 2004), geNorm (Vandesompele et al., 2002), and BestKeeper (Pfaffl et al., 2004). BestKeeper was designed to analyze a maximum of 10 genes at one time. Therefore, the 10 genes with the lowest variation of cycle threshold $(\mathbf{C t})$ values were used in analysis of the BestKeeper correlation coefficient $(\mathbf{r})$.

All analyses were performed by all 3 methods to provide information on stability in the different physi- 
ologic states: (1) all samples, (2) samples during pregnancy, and (3) samples during regression, consisting of samples from the late luteal phase, and early and late regression.

The importance of reference gene selection was illustrated by 2 analyses. First, 3 target genes [steroidogenic acute regulatory protein $(S T A R)$, cytochrome P450 family 11 subfamily A member 1 (CYP11A1), and prostaglandin $\mathrm{F}$ receptor $(P T G F R)$; primers listed in Supplemental Table S1, https://doi.org/10.3168/ jds.2019-17526] were analyzed by normalization with $A C T B$ and $G A P D H$ or normalized with a pair of reference genes based on results from NormFinder or geNorm algorithms using the delta delta ct method (Livak and Schmittgen, 2001). Second, the 3 target genes were normalized using the geometric mean of all 15 analyzed stable genes to produce a "silver standard" (Pfaffl et al., 2004). Then, $\mathrm{r}$ were calculated by comparing values normalized to the 3 different pairs of reference genes with the values from the silver standard. In addition, the absolute value of the deviation from the silver standard was calculated for each sample. The average deviation using each pair of reference genes was then compared by ANOVA analysis in PROC MIXED (SAS version 9.4, SAS Institute Inc., Cary, NC) to identify whether reference genes used for normalization affected variation from the silver standard.

\section{RESULTS}

\section{Identification and Consideration of Reference Genes Used in Recent Literature}

In total, 20 reference genes were identified in papers published within the last 5 yr (Supplemental Table S2, https://doi.org/10.3168/jds.2019-17526). Of these, 7 were highly differentially expressed $(\mathrm{Q}<0.01)$ in the present RNA-seq study in the late regression versus late luteal phase comparison. Of note was $G A P D H$, with a 1.48-fold increase in late regression $(\mathrm{Q}=0.0219$, Supplemental Table S2).

\section{Proposed New Reference Genes}

Stable reference genes were determined using both an ERCC-corrected and a FPKM-corrected data set. One gene was identified in both lists: ZNF266, and was therefore selected for further validation. From the FPKM-corrected data set, 6 genes met the following combination of criteria for selection: those with a base mean $>50$, ANOVA $P$-value $>0.45$, and DEseq 2 $P$-value $>0.45$. These were CDC42, EIF3E, HIF1A, CD63, YWHAE, and PPIB. From the FPKM-corrected data set, 7 additional genes were selected which met the criteria that DESeq2 $P$-values were $>0.80: R P L 4$, UQCRFS1, COX 4I1, RPS4X, SSR3, RPL22, and CST3. However, RPL22 was not pursued because within attempted validation of 4 primer pairs the average $\mathrm{Ct}$ value was $36.16 \pm 1.02$, thus expression was too low to serve as a robust reference gene.

This resulted in 13 genes for further stability analysis (Supplemental Table S3, https://doi.org/10.3168/ jds.2019-17526), in addition to $A C T B$ and GAPDH (Supplemental Table S2). Additional stable genes were not further validated (Supplemental Tables S4 and S5, https://doi.org/10.3168/jds.2019-17526), consisting largely of ribosomal subunits (33\% of additional stable genes). Pathway analysis of stable genes yielded 35 significant terms $(P<0.05), 12$ of which were nonredundant, having a unique set of input genes and distinct biological processes. These included processes involved in cellular metabolism, ATP production, and protein synthesis (Supplemental Table S6, https://doi.org/10 $.3168 /$ jds.2019-17526).

\section{PCR Validation of Proposed Reference Genes in Samples Collected from All Physiologic States}

Stability rankings in geNorm (Figure 1) and NormFinder (Figure 2) consistently found the same 3 genes as most stable: RPS $4 X, C O X 4 I 1$, and PPIB, and the same 4 as least stable: CST3, ZNF266, GAPDH, and $U Q C R F S$, though the exact ranking was not the same in each algorithm. The average difference in ranking between the 2 algorithms was $1.1 \pm 0.21$, with $80 \%$ (12 of 15) of the genes less than one position away from the position of that gene using the other algorithm.

The geNorm provides $\mathrm{V}$ values to determine the benefit of adding additional reference genes to an experiment. In this data set, using the cutoff of 0.15 as recommended in the original publication (Vandesompele et al., 2002), use of the 2 most stable genes (RPS $4 X$ and $C O X_{4} I 1$ ) was sufficient for normalization (Figure 1B). NormFinder calculates stability for pairs of reference genes and recommended SSR 3 and $C D C 42$.

BestKeeper provides several parameters for evaluation, not a single aggregate value to rank genes. In the original publication, it was recommended that potential reference genes have a standard deviation of Ct values below 1, criteria which all evaluated genes met (Table 1). Additionally, it was recommended in the original publication that genes with a higher $\mathrm{r}$ with the BestKeeper Index are more stable. Of the genes evaluated, all had $\mathrm{r}$ between 0.91 and 0.99 , with the top 5 genes $\geq 0.98$, indicating all potential new reference genes evaluated are reasonably stable within the CL. When considering the top 3 genes identified by geNorm 
A. GeNorm Stability: All Samples

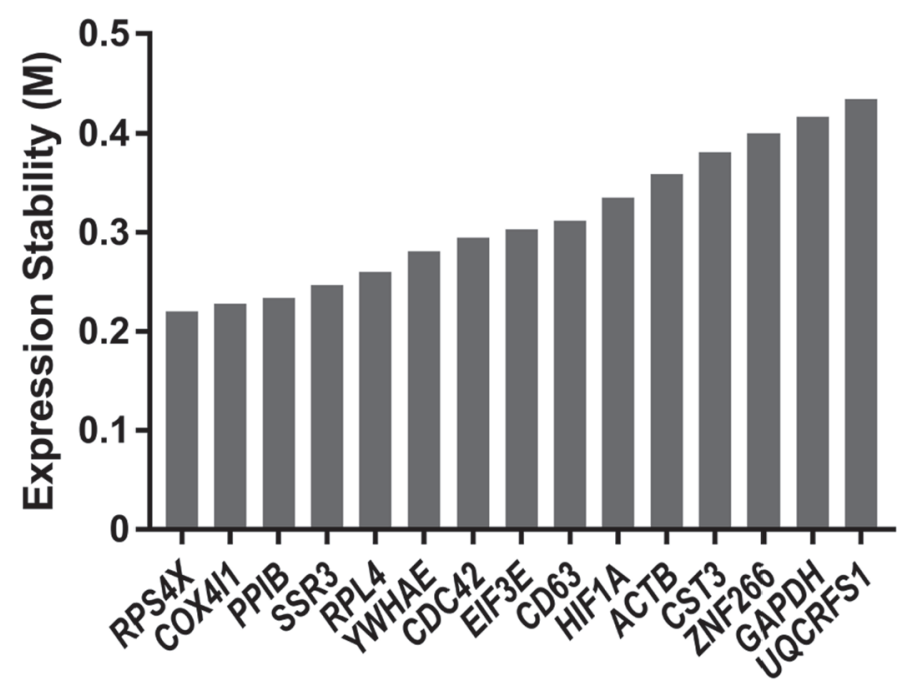

B. GeNorm V: All Samples

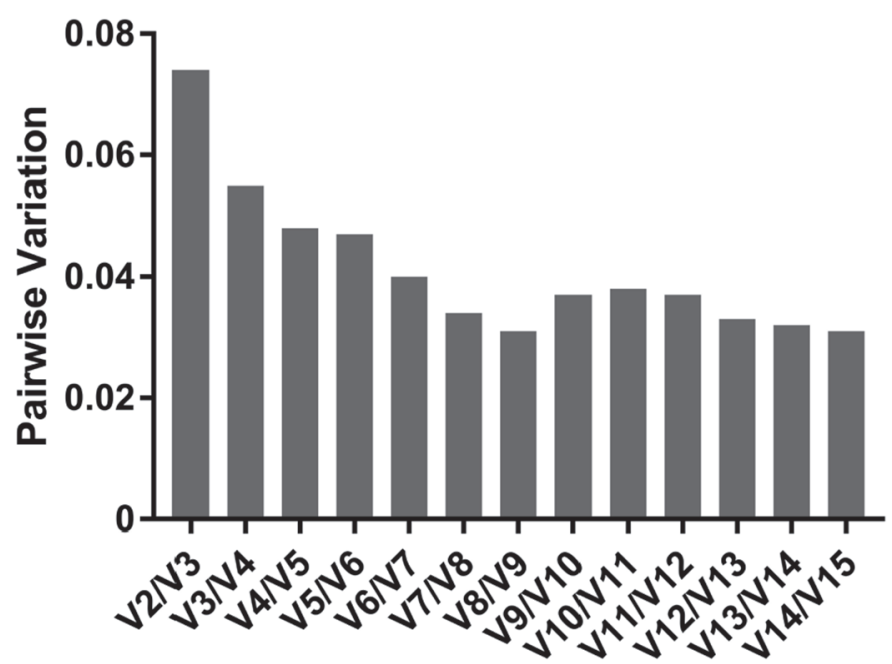

Figure 1. For analysis of all samples: (A) geNorm (Vandesompele et al., 2002) M values, where lower values represent more stable genes, and (B) geNorm $\mathrm{V}$ values. Once $\mathrm{V}$ values are below the cut off of 0.15 , adding additional reference genes provides little benefit.

and NormFinder, these had a high BestKeeper correlation coefficient if they were in the 10 genes evaluated, although not the lowest $\mathrm{Ct}$ variance (Table 1). The variance in $\mathrm{Ct}$ value noted by BestKeeper was also reflected in the box and whisker plot of $\mathrm{Ct}$ values for individual samples (Figure 3).

\section{RT-qPCR Validation of Proposed Reference Genes in Samples Collected During Luteolysis}

Gene rankings in NormFinder and geNorm were more consistent in regression than in pregnancy alone, but less consistent than with all samples, with an average difference in rankings of $1.7 \pm 0.3$ between algorithms. The same gene was identified as most stable in both algorithms: $R P S 4 X$ (Figure 4). Calculations using geNorm recommended RPS $4 X$ and COX $I_{11}$ for normalization, whereas NormFinder recommended $R P L 4$ and SSR3.

BestKeeper analyses resulted in $r$ to the BestKeeper index $>0.92$ for the 10 genes analyzed, with standard deviation of $\mathrm{Ct}$ values below 0.91 for the top 10 genes. However, Ct standard deviation exceeded 1 for both GAPDH and UQCRFS1, values above the cut-off for stable genes (Table 1). Thus, for regression the same genes recommended when all samples are considered are close to optimal for use in CL regression.

\section{$R T$-qPCR Validation of Proposed Reference Genes in Samples Collected During Pregnancy}

Gene rankings in NormFinder and geNorm were the least consistent in pregnancy relative to other analyses, with average difference in rankings between the algorithms $2.4 \pm 0.4$. The top 3 genes between algorithms had only 1 shared gene. The top 3 most stable genes in geNorm were $C D C 42, Y W H A E$, and $C D 63$, whereas NormFinder identified PPIB, CDC42, and CST3 (Figure 5). The geNorm calculations recommended $C D C 42$ and $Y W H A E$ for normalization, whereas NormFinder recommended $C D C 42$ and $P P I B$.

BestKeeper analysis calculated the variation in $\mathrm{Ct}$ values to be lower in pregnancy than in cycling animals or all samples, and all were under 0.7. Correlations with

\section{NormFinder Stability: All Samples}

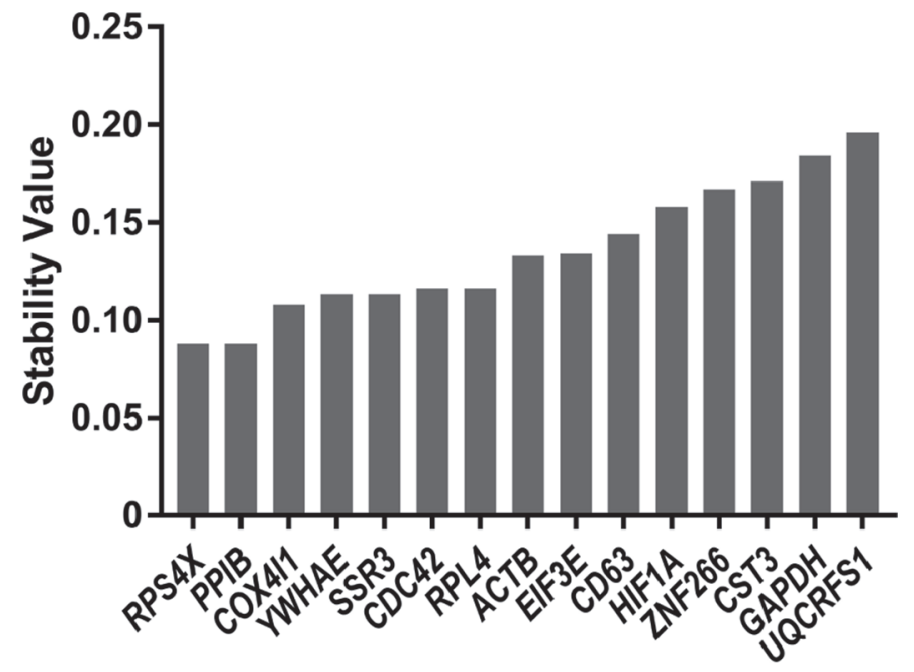

Figure 2. NormFinder (Andersen et al., 2004) stability values with all samples in analysis; the lower the value, the more stable the gene. 
the BestKeeper Index were high in analyzed genes, with the lowest $\mathrm{r}$ equal to 0.91 (Table 1 ).

Analyses indicated genes found to be most stable in regression and analysis of all samples were still more stable than traditional reference genes in pregnancy. However, a distinct difference was observed in optimal reference genes for pregnancy, with $C D C 42$ being the most stable based on the combination of geNorm and NormFinder results.

\section{Comparison of Gene Stability Among Physiological Stages}

Most genes evaluated met the criteria set out in the original publication of BestKeeper, with the exception of GAPDH and UQCRFS1 in regression. NormFinder and geNorm provided the ability to rank genes, and reliably ranked several genes in the bottom $30 \%$ of genes regardless of physiologic condition: GAPDH, UQCRFS1, and ZNF266. Less consistency was observed in the most stable genes, particularly in pregnancy alone, where $R P S 4 X$ went from first in regression and all samples to 10th in pregnancy and CST3, which went from 13th with all samples and 14th in regression to third most stable in NormFinder analyses. This trend was repeated in geNorm where $R P S 4 X$ went from first in all and regression to 11th in pregnancy, though CST3 underwent a less dramatic change in pregnancy, where it went from 12th in all samples and 13th in regression to 7 th in pregnancy.

\section{Effect of Reference Gene Selection on Target Gene Analyses}

Three target genes (STAR, PTGFR, and CYP11A1) were analyzed with different pairs of reference genes

\section{Ct Values of Genes}

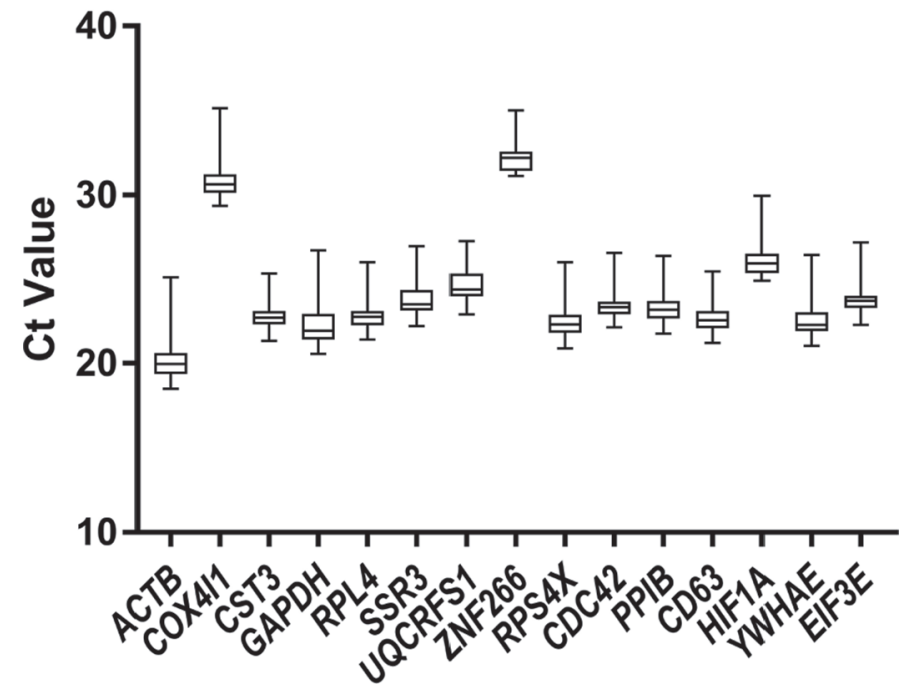

Figure 3. Box and whisker plot of cycle threshold $(\mathrm{Ct})$ values of all samples for each gene. The middle line represents the second quartile, the box ends represent the first and third quartiles, and whiskers represent maximum and minimum values.

to illustrate the effect of reference gene selection. Use of individual reference genes for normalization was not considered, as use of a single gene has been shown to be suboptimal (Vandesompele et al., 2002). Three reference gene pairs were selected for calculation of fold change relative to the first month of pregnancy: (1) traditional genes $A C T B$ and GAPDH, (2) the most stable pair using Norm Finder: SSR3 and CDC42, and (3) the top 2 stable genes in geNorm after COX $4 I 1$ was removed: PPIB and RPS4X. The COX $4 I 1$ was removed due to low expression in CL (Ct values $\sim 9$ cycles greater than target genes).

Table 1. BestKeeper (Pfaffl et al., 2004): SD of cycle threshold (Ct) and $\mathrm{r}$ to BestKeeper Index

\begin{tabular}{|c|c|c|c|c|c|c|}
\hline \multirow[b]{2}{*}{ Gene } & \multicolumn{2}{|c|}{ All samples } & \multicolumn{2}{|c|}{ Regression } & \multicolumn{2}{|c|}{ Pregnancy } \\
\hline & Ct SD & $\mathrm{r}$ & Ct SD & $\mathrm{r}$ & Ct SD & $\mathrm{r}$ \\
\hline$A C T B$ & 0.80 & - & 0.96 & - & 0.50 & - \\
\hline$C D 63$ & 0.57 & 0.97 & 0.69 & 0.98 & 0.29 & 0.95 \\
\hline$C D C 42$ & 0.52 & 0.99 & 0.62 & 0.99 & 0.33 & 0.98 \\
\hline COX4I1 & 0.74 & - & 0.93 & - & 0.39 & - \\
\hline CST3 & 0.56 & 0.92 & 0.67 & 0.93 & 0.31 & 0.97 \\
\hline EIF3E & 0.56 & 0.98 & 0.73 & 0.98 & 0.27 & 0.91 \\
\hline$G A P D H$ & 0.91 & - & 1.07 & - & 0.62 & - \\
\hline$H I F 1 A$ & 0.72 & 0.93 & 0.91 & 0.94 & 0.37 & 0.97 \\
\hline$P P I B$ & 0.66 & 0.97 & 0.82 & 0.97 & 0.34 & 0.99 \\
\hline$R P L 4$ & 0.57 & 0.99 & 0.71 & 0.99 & 0.27 & 0.97 \\
\hline$R P S 4 X$ & 0.69 & 0.98 & 0.85 & 0.99 & 0.41 & - \\
\hline SSR3 & 0.74 & - & 0.94 & - & 0.37 & 0.96 \\
\hline UQCRFS1 & 0.83 & - & 1.05 & - & 0.42 & - \\
\hline$Y W H A E$ & 0.69 & 0.99 & 0.86 & 0.99 & 0.36 & 0.98 \\
\hline ZNF266 & 0.61 & 0.91 & 0.75 & 0.92 & 0.3 & 0.91 \\
\hline
\end{tabular}


A. GeNorm Stability: Regression

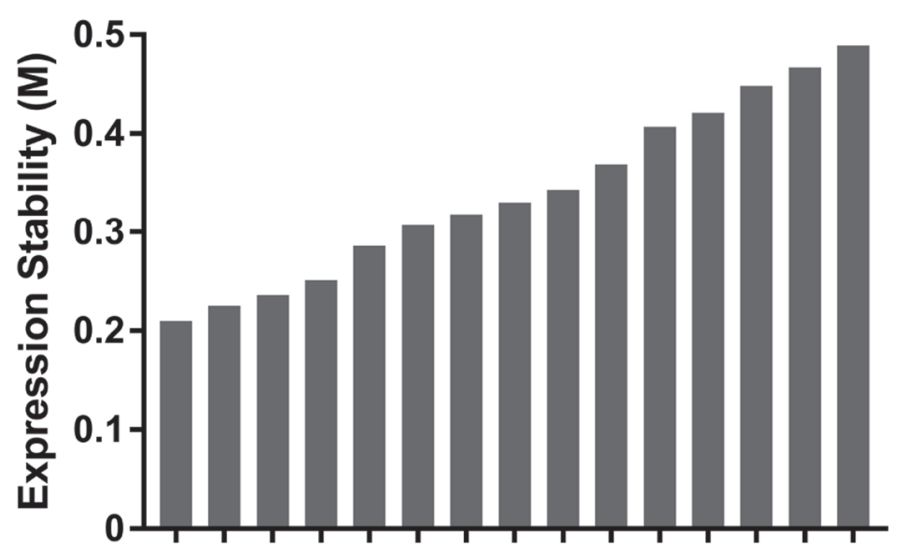

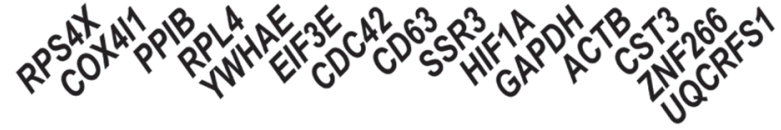

B. NormFinder Stability: Regression
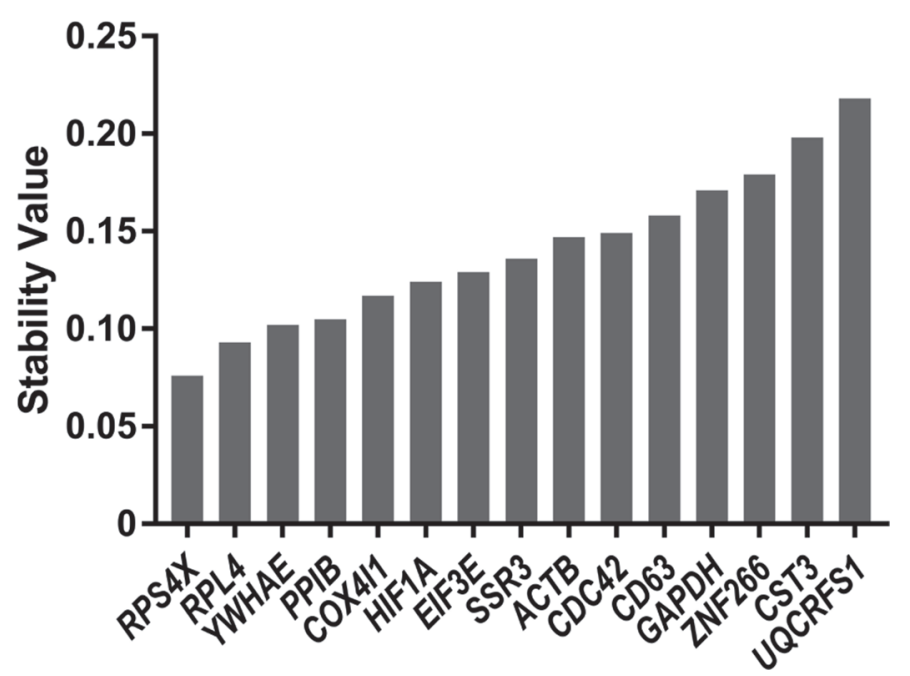

Figure 4. Gene stability in samples collected throughout luteolysis. Analysis includes samples from the late luteal phase [d 18 of cycle, no decline in progesterone (P4) following $24 \mathrm{~h}$ ], early regression [exposed to at least one $\mathrm{PGF}_{2 \alpha}$ metabolite (PGFM) pulse, but no significant decline in P4], and late regression $(\mathrm{P} 4<1 \mathrm{ng} / \mathrm{mL})$ : (A) geNorm (Vandesompele et al., 2002) stability and (B) NormFinder (Andersen et al., 2004) stability.

Use of different reference gene pairs resulted in different statistical interpretation of the data in at least one comparison within all 3 target genes. For example, $S T A R$, was statistically different in the late luteal phase from first month of pregnancy when normalized with $P P I B$ and $R P S 4 X$ or SSR3 and $C D C 42$ but was not different when normalized with $G A P D H$ and $A C T B$. Similar differences in significance between normalization with $G A P D H$ and $A C T B$ versus $P P I B$ and
$R P S 4 X$ or $S S R 3$ and $C D C 42$ were noted for STAR in the second month of pregnancy, for PTGFR in early regression, and for $C Y P 11 A 1$ in both early regression and late luteal phase. Thus, use of different reference genes for normalization, even when 2 reference genes were used, resulted in differences in interpretation of results for all 3 of the genes that were evaluated in this analysis.

To further quantify the effect of reference gene selection on analyses, the 3 target genes were normalized using a "silver standard" based on all 15 reference genes. Correlation with the silver standard was greater

\section{A. GeNorm Stability: Pregnancy}

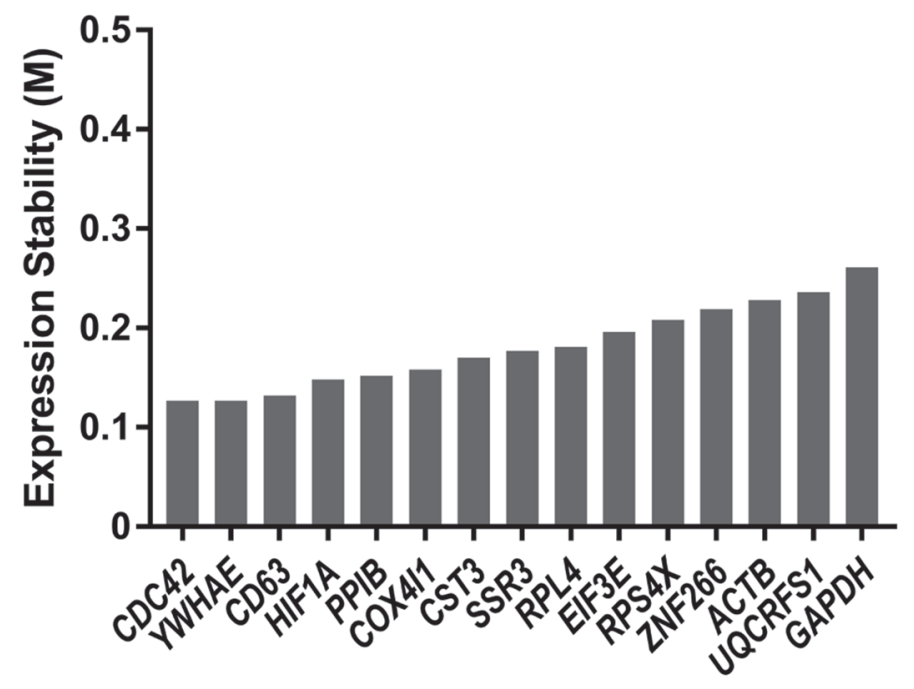

B. NormFinder Stability: Pregnancy

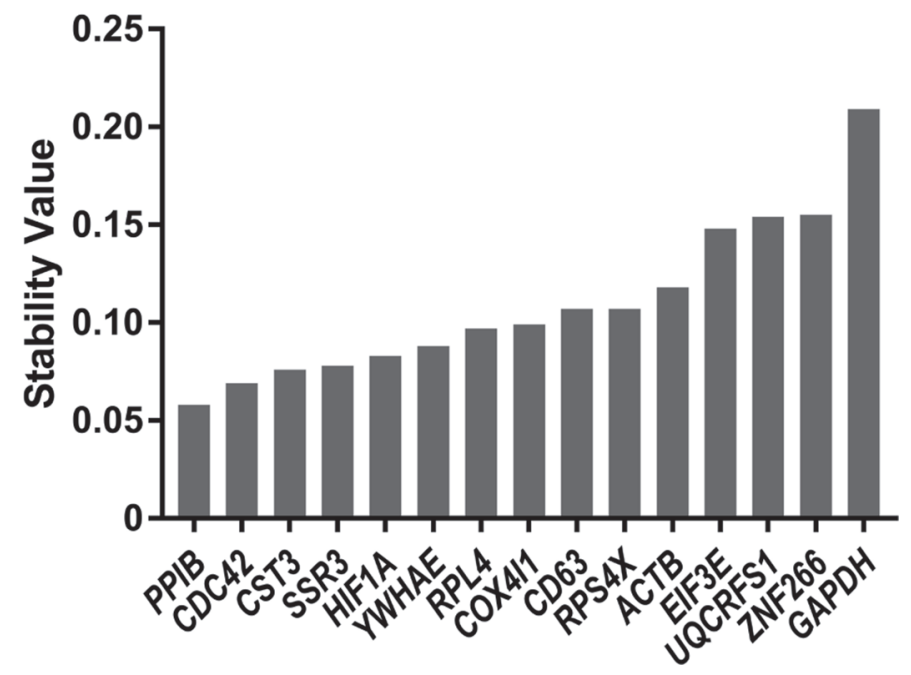

Figure 5. Analysis of samples in the first and second months of pregnancy: (A) geNorm (Vandesompele et al., 2002) stability and (B) NormFinder (Andersen et al., 2004) stability. 
for the combination of $P P I B$ and $R P S 4 X$ as well as SSR 3 and CDC42 than ACTB and GAPDH (Table 2). Additionally, the residual of the fold change from the silver standard (calculated as the absolute value of the fold change when pair was used minus the fold change when silver standard was used) was more than 3 -fold greater $(P<0.05)$ for $S T A R$ and $C Y P 11 A 1$ and more than 2-fold greater $(P<0.05)$ for PTGFR when values were normalized with $A C T B$ and $G A P D H$ compared with values when normalized with $P P I B$ and $R P S_{4} X$ or SSR3 and CDC42 (Table 2).

\section{DISCUSSION}

Studies of luteal physiology, including the molecular processes that control CL regression or maintenance during pregnancy, are critical for designing optimized reproductive programs and for developing methods to decrease pregnancy loss and optimize circulating P4 concentrations. Thus, it is concerning that $35 \%$ of previously used reference genes are observed to be differentially expressed when late regression CL are compared with those from the late luteal phase (Supplemental Table S2, https://doi.org/10.3168/jds.2019-17526). No one gene is stable across every study question, physiologic state, breed, or disease, and thus every study requires validation of reference genes used and the following of best practices such as utilization of more than one reference gene (Vandesompele et al., 2002). However, this report provides a novel set of stable genes as a starting point for identification of valid reference genes for future experiments. A total of 74 potentially stable genes were identified using data from whole transcriptome sequencing (Table 2, Supplemental Tables S2 and S3, https://doi.org/10.3168/jds.2019-17526). Of these, 13 genes were selected for stability analysis via RTqPCR and compared with 2 traditional reference genes ( $A C T B$ and $G A P D H)$ in an independent set of samples. In accordance with the goal of the experiment, 7 genes were identified with greater stability than $A C T B$ and $G A P D H$ in all physiologic states investigated: $R P S 4 X$, COX4I1, PPIB, SSR3, RPL4, YWHAE, and CDC42, with another 3 identified for use during pregnancy: CST3, HIF1A, and CD63. The importance of reference gene selection was then illustrated by displaying the effect of normalization to $R P S 4 X$ and $P P I B$ or $S S R 3$ and $C D C 42$ compared with $G A P D H$ and $A C T B$.

\section{Predicted Functional Roles of Stably Expressed Genes}

Of the stable genes identified, by far the largest functional class present were genes encoding ribosomal protein subunits ( $32 \%$ of identified stable genes), subunits
Table 2. Correlation coefficient of the silver standard of select pairs of reference genes

\begin{tabular}{lccc}
\hline Item & STAR & CYP11A1 & PTGFR \\
\hline$A C T B$ and GAPDH & & & \\
$\quad$ Correlation & 0.879 & 0.909 & 0.759 \\
$\quad$ Residual $( \pm$ SEM) & $0.11 \pm 0.03^{\mathrm{a}}$ & $0.11 \pm 0.02^{\mathrm{a}}$ & $0.14 \pm 0.04^{\mathrm{a}}$ \\
$\begin{array}{c}\text { SSR3 and CDC42 } \\
\text { Correlation }\end{array}$ & 0.993 & 0.980 & 0.984 \\
Residual $( \pm$ SEM) & $0.03 \pm 0.01^{\mathrm{b}}$ & $0.03 \pm 0.01^{\mathrm{b}}$ & $0.05 \pm 0.1^{\mathrm{b}}$ \\
$\begin{array}{l}\text { PPIB and } R P S 4 X \\
\text { Correlation }\end{array}$ & 0.993 & 0.990 & 0.966 \\
Residual $( \pm$ SEM) & $0.02 \pm 0.01^{\mathrm{b}}$ & $0.02 \pm 0.01^{\mathrm{b}}$ & $0.03 \pm 0.01^{\mathrm{b}}$ \\
\hline
\end{tabular}

${ }^{\mathrm{a}, \mathrm{b}}$ Values within a column with different superscripts differ $(P<0.05)$.

of the mitochondrial ATP synthase ( $10 \%$ of genes), and proteins involved in the electron transport chain (8\%). The near constant expression of these genes was consistent with the vital role of ribosomes/protein synthesis and mitochondria/ATP generation in the cell (Koonin, 2000).

When considering the function of the genes selected for further evaluation with RTq-PCR, most of the encoded proteins had functions that would be predicted to be stable. These included proteins with a role in the mitochondrial electron transport chain, ribosomal subunits, protein processing and transport, cell cycle control, and a rho GTPase (Fisher et al., 1990; van Hemert et al., 2001; Kim et al., 2008; Pols and Klumperman, 2009; Li et al., 2011; Melendez et al., 2011; Pyott et al., 2011; Rufini et al., 2012; Leal et al., 2016; Bottani et al., 2017; Huber and Hoelz, 2017; Pfeffer et al., 2017; Sesen et al., 2017).

An exception to this trend is HIF1A and CST3. The $H I F 1 A$ is a transcription factor activated by exposure to reductions in oxygen (Bunn and Poyton, 1996) and hormonally regulated by human chorionic gonadotropin in human CL, and thus presumably regulated by luteinizing hormone as well (van den Driesche et al., 2008), whereas CST3 is an inhibitor of lysosomal cysteine proteases, which has been characterized in the ovine uterus where expression appears to be regulated by progesterone and interferon tau (Song et al., 2006). Regulation of HIF1A and CST3 in other species would not have led to predictions of stability, and suggests stability observed in the present study is related to the physiologic stage and environment of the CL. Thus, they may not be stable outside of the physiologic considerations evaluated. Indeed, CST3 experiences a substantial fall in stability ranking in samples during CL regression compared with pregnancy (stability ranking in pregnancy vs. regression alone analyses: NormFinder: 3rd to 14th, geNorm: 7th to 13th), which may be related to the fall in $\mathrm{P} 4$ production at this time. Therefore, whereas these genes may have been 
more stable than traditional reference genes in the present analyses, our results emphasize the importance of physiologic stage and of validating reference genes in each experimental condition, especially where there is a possibility of pregnancy loss or CL regression.

\section{Presence of Identified Reference Genes in Previous Nonbovine Studies}

Stable genes were identified through transcriptomic analysis, rather than consideration of gene function or historic use in normalization. Despite this, of the 13 genes evaluated for stability via RTq-PCR, $69 \%$ had previously been evaluated as candidate reference genes for RT-qPCR in diverse species from zebra fish to rats with 4 of these genes having been identified through RNA-seq or microarray (Nygard et al., 2007; Hoogewijs et al., 2008; Popovici et al., 2009; Too and Ling, 2011; Mossberg et al., 2016; Xu et al., 2016; Samiullah et al., 2017; Malik and George, 2018; Shaydurov et al., 2018). Additionally, COX $4 I 1$ has been evaluated for use as a control in western blots (Lee et al., 2016) and HIF1A has a housekeeping-like promoter noted in data from adult mouse tissues (Wenger et al., 1998). That overall $85 \%$ of genes investigated in this study using an objective transcriptomic approach had been previously considered for use in normalization using a combination of transcriptomic and non-transcriptomic approaches suggests either method may identify stably transcribed genes but that a genome-wide, whole-transcriptome approach is a highly efficient tool in screening for stably expressed genes.

Of note in the stable gene list is the absence of $G A P$ $D H$ and $A C T B$. Though this is worth highlighting due to the prevalence of $G A P D H$ and $A C T B$ in RT-qPCR normalization, this is not a surprise as both $G A P D H$ and $A C T B$ have been known for almost $20 \mathrm{yr}$ to be unstable in some tissues and conditions (Suzuki et al., 2000). Despite this, GAPDH and ACTB continue to be the most common genes used for normalization in the CL without additional validation of stability. This is troubling because in our analyses GAPDH ranged from 11th to 15 th in stability, being the least stable gene by both algorithms in CL from pregnant animals, and the 2nd least stable when all samples were considered. Furthermore, by RNA-seq analysis, GAPDH was upregulated in CL tissue in late regression (circulating $\mathrm{P} 4<1 \mathrm{ng} / \mathrm{mL}$ ) compared with late luteal phase tissue (Supplemental Table S2, https://doi.org/10.3168/jds .2019-17526), making it particularly unfit as a reference gene for tissues that may be undergoing luteolysis. The other common reference gene, $A C T B$, is more stable than $G A P D H$, though $A C T B$ was consistently ranked 8th to 13th depending on algorithm and conditions, putting it within the lower half of stability rankings, and indicating that there are viable options for normalization more stable than $A C T B$.

\section{Influence of Algorithmic and Technical Differences}

Throughout analyses differences were observed in rankings by geNorm and NormFinder, though the same RT-qPCR data were used in both algorithms. Fundamental differences in algorithms are likely responsible for inconsistences, as NormFinder takes into account intergroup and intragroup variation, whereas geNorm calculations are based solely on variation among all samples (Andersen et al., 2004). Because geNorm analyzes variation among samples, calculations are strongly influenced by coregulation of genes, which may have biased results in the current study where more than one gene from the same pathway is present as genes were selected by calculation, not by hand picking genes from independent pathways. Thus, it was especially important to validate geNorm results through NormFinder and BestKeeper algorithms in the present study, and future work needs to account for this source of bias if using geNorm to validate stable genes from this study for novel experimental conditions. Number of samples in the analysis may also have affected the ability of the algorithms to determine stability, as there was a correlation of number of samples in analysis and consistency between algorithms, with the largest group (all) being most consistent, and the smallest group (pregnancy alone) least consistent between the algorithms. Despite these differences, the most and least stable genes remained consistent in the "regression" and "all" analyses. Greater differences would be noted if one compared geNorm and NormFinder rankings to the order of the BestKeeper index $r$ and the standard deviation of $\mathrm{Ct}$ values for each gene than when geNorm and NormFinder are compared. However, this analysis was not undertaken as BestKeeper was not developed as a method to provide a single calculated stability value and ranking, but rather to report several parameters for each gene which can be used to determine if that gene may be suitable for normalization (Pfaffl et al., 2004).

RNA-seq allowed for identification of novel bovine CL reference genes, although the genes with the highest $P$-value in RNA-seq analyses were not the same as those indicated to be most stable in PCR analyses. This is likely due to a combination of differences in algorithms used between RNA-seq and RT-qPCR and differences in samples used. In the RNA-seq analysis, genes were evaluated for stability based on p-values from ANOVA and DEseq2 analyses, whereas RT-qPCR analyses were based on analysis of variation between 
samples. Some variation is also expected between the different set of samples for RT-qPCR and RNA-seq, though this is likely a smaller influence than that seen from algorithmic differences.

Thus, due to differences in statistical methodology and independent samples, it is unsurprising that results are not identical. Despite these differences, RNA-seq still provided a practical method of identifying novel reference genes, avoiding the subjectivity of gene selection inherent in other approaches to identify genes for stability analysis. Nevertheless, RT-qPCR validation may still be important before utilization of a new reference gene for normalization.

\section{CONCLUSIONS}

Overall, this study demonstrates the need for careful evaluation of reference gene expression stability during studies of reproductive physiology in cattle and specifically in studies of the CL, with the most dramatic differences observed in gene stability during CL regression and pregnancy. Despite the dynamic nature of the CL, 7 new genes were identified as suitable reference genes, with another 3 in CL from pregnant animals. Many of the identified genes had previously been used in other species and tissues, indicating the efficiency of the whole transcriptome sequencing approach. Still, it is of note that optimal reference genes in pregnancy are not precisely the same as those in regression. Thus, RNA-seq data can be used not only for identifying differentially expressed genes but also for identifying stable reference genes. Although no single set of genes is stable in all experimental conditions, requiring validation of reference genes for each RT-qPCR experiment, this report provides an objective reference for selection of genes to validate in future studies.

\section{ACKNOWLEDGMENTS}

Thanks to the animal care staff at the University of Wisconsin-Madison Dairy Cattle Center, as well as to Caio A. Gamarra, Rodrigo S. Gennari, Victor E. Gomez-Leon, and Rafael R. Domingues (Department of Dairy Science, University of Wisconsin, Madison) for help with original collection of CL biopsies. The authors declare that the research was conducted in the absence of any commercial or financial relationships that could be construed as a potential conflict of interest. Research was supported by BARD-US Israel Binational Agricultural Research and Development Fund grant IS-4799-15 to MCW and Wisconsin Experiment Station as Hatch Project WIS01240 to MCW. Wenli Li was supported by appropriated project 5090-31000-026-00-D from the USDA Agriculture Research Service (Dairy Forage Re- search Center, Madison, WI). Mention of trade names or commercial products in this article is solely for the purpose of providing specific information and does not imply recommendation by the US Department of Agriculture. The USDA is an equal opportunity provider and employer.

\section{REFERENCES}

Anders, S., P. T. Pyl, and W. Huber. 2015. HTSeq-A Python framework to work with high-throughput sequencing data. Bioinformatics 31:166-169. https://doi.org/10.1093/bioinformatics/btu638.

Andersen, C. L., J. L. Jensen, and T. F. Orntoft. 2004. Normalization of real-time quantitative reverse transcription-PCR data: A model-based variance estimation approach to identify genes suited for normalization, applied to bladder and colon cancer data sets. Cancer Res. 64:5245-5250. https://doi.org/10.1158/0008-5472 .CAN-04-0496.

Bas, A., G. Forsberg, S. Hammarstrom, and M. L. Hammarstrom. 2004. Utility of the housekeeping genes $18 \mathrm{~S}$ rRNA, beta-actin and glyceraldehyde-3-phosphate-dehydrogenase for normalization in real-time quantitative reverse transcriptase-polymerase chain reaction analysis of gene expression in human T lymphocytes. Scand. J. Immunol. 59:566-573. https://doi.org/10.1111/j.0300-9475.2004 $.01440 . x$.

Bottani, E., R. Cerutti, M. E. Harbour, S. Ravaglia, S. A. Dogan, C. Giordano, I. M. Fearnley, G. D'Amati, C. Viscomi, E. FernandezVizarra, and M. Zeviani. 2017. TTC19 plays a husbandry role on UQCRFS1 turnover in the biogenesis of mitochondrial respiratory complex III. Mol. Cell 67:96-105. https://doi.org/10.1016/j.molcel 2017.06.001.

Bunn, H. F., and R. O. Poyton. 1996. Oxygen sensing and molecular adaptation to hypoxia. Physiol. Rev. 76:839-885. https://doi.org/ 10.1152/physrev.1996.76.3.839

Chen, J., E. E. Bardes, B. J. Aronow, and A. G. Jegga. 2009. ToppGene Suite for gene list enrichment analysis and candidate gene prioritization. Nucleic Acids Res. 37(Web Server):W305-W311. https://doi.org/10.1093/nar/gkp427.

Chen, L., F. H. Sun, X. D. Yang, Y. L. Jin, M. K. Shi, L. Wang, Y. Shi, C. Zhan, and Q. Wang. 2017. Correlation between RNASeq and microarrays results using TCGA data. Gene 628:200-204. https://doi.org/10.1016/j.gene.2017.07.056.

de Jonge, H. J. M., R. S. N. Fehrmann, E. de Bont, R. M. W. Hofstra, F. Gerbens, W. A. Kamps, E. G. E. de Vries, A. G. J. van der Zee, G. J. te Meerman, and A. ter Elst. 2007. Evidence based selection of housekeeping genes. PLoS One 2:e898. https://doi.org/10.1371/ journal.pone.0000898.

Fabregat, A., S. Jupe, L. Matthews, K. Sidiropoulos, M. Gillespie, P. Garapati, R. Haw, B. Jassal, F. Korninger, B. May, M. Milacic, C. D. Roca, K. Rothfels, C. Sevilla, V. Shamovsky, S. Shorser, T. Varusai, G. Viteri, J. Weiser, G. M. Wu, L. Stein, H. Hermjakob, and P. D'Eustachio. 2018. The reactome pathway knowledgebase. Nucleic Acids Res. 46(D1):D649-D655. https://doi.org/10.1093/ nar/gkx1132.

Fisher, E. M. C., P. Beer-Romero, L. G. Brown, A. Ridley, J. A. McNeil, J. B. Lawrence, H. F. Willard, F. R. Bieber, and D. C. Page. 1990. Homologous ribosomal protein genes on the human X and Y chromosomes: Escape from X inactivation and possible implications for Turner syndrome. Cell 63:1205-1218. https://doi.org/10 .1016/0092-8674(90)90416-C.

Glare, E. M., M. Divjak, M. J. Bailey, and E. H. Walters. 2002. Betaactin and GAPDH housekeeping gene expression in asthmatic airways is variable and not suitable for normalising mRNA levels. Thorax 57:765-770. https://doi.org/10.1136/thorax.57.9.765.

Hoogewijs, D., K. Houthoofd, F. Matthijssens, J. Vandesompele, and J. R. Vanfleteren. 2008. Selection and validation of a set of reliable reference genes for quantitative sod gene expression analysis in C-elegans. BMC Mol. Biol. 9:9. https://doi.org/10.1186/1471 $-2199-9-9$ 
Huber, F. M., and A. Hoelz. 2017. Molecular basis for protection of ribosomal protein L4 from cellular degradation. Nat. Commun. 8:14354. https://doi.org/10.1038/ncomms14354.

Huggett, J., K. Dheda, S. Bustin, and A. Zumla. 2005. Real-time RTPCR normalisation; strategies and considerations. Genes Immun. 6:279-284. https://doi.org/10.1038/sj.gene.6364190.

Kim, D., G. Pertea, C. Trapnell, H. Pimentel, R. Kelley, and S. L. Salzberg. 2013. TopHat2: Accurate alignment of transcriptomes in the presence of insertions, deletions and gene fusions. Genome Biol. 14:R36. https://doi.org/10.1186/gb-2013-14-4-r36.

Kim, J., T. G. Choi, Y. Ding, Y. Kim, K. S. Ha, K. H. Lee, I. Kang, J. Ha, R. J. Kaufman, J. Lee, W. Choe, and S. S. Kim. 2008. Overexpressed cyclophilin B suppresses apoptosis associated with ROS and Ca2+ homeostasis after ER stress. J. Cell Sci. 121:3636-3648. https://doi.org/10.1242/jcs.028654.

Koonin, E. V. 2000. How many genes can make a cell: The minimalgene-set concept. Annu. Rev. Genomics Hum. Genet. 1:99-116. https://doi.org/10.1146/annurev.genom.1.1.99.

Langmead, B., and S. L. Salzberg. 2012. Fast gapped-read alignment with Bowtie 2. Nat. Methods 9:357-359. https://doi.org/10.1038/ nmeth.1923.

Leal, M. F., H. F. Ribeiro, J. A. Rey, G. R. Pinto, M. C. Smith, C. A. Moreira-Nunes, P. P. Assumpcao, L. M. Lamarao, D. Q. Calcagno, R. C. Montenegro, and R. R. Burbano. 2016. YWHAE silencing induces cell proliferation, invasion and migration through the up-regulation of $\mathrm{CDC} 25 \mathrm{~B}$ and $\mathrm{MYC}$ in gastric cancer cells: New insights about YWHAE role in the tumor development and metastasis process. Oncotarget 7:85393-85410. https://doi.org/10 .18632 /oncotarget.13381.

Lee, H. G., J. Jo, H. H. Hong, K. K. Kim, J. K. Park, S. J. Cho, and C. Park. 2016. State-of-the-art housekeeping proteins for quantitative western blotting: Revisiting the first draft of the human proteome. Proteomics 16:1863-1867. https://doi.org/10.1002/pmic 201500344.

Li, X. B., J. Chen, M. Deng, F. Wang, Z. Du, and J. Zhang. 2011. Zinc finger protein HZF1 promotes K562 cell proliferation by interacting with and inhibiting INCA1. Mol. Med. Rep. 4:1131-1137.

Livak, K. J., and T. D. Schmittgen. 2001. Analysis of relative gene expression data using real-time quantitative PCR and the 2- $\Delta \Delta \mathrm{CT}$ method. Methods 25:402-408. https://doi.org/10.1006/meth.2001 .1262 .

Malik, N., and J. K. George. 2018. Resistance genes in piper colubrinum: In silico survey from leaf transcriptome and expression studies upon challenge inoculation with phytophthora capsici. Appl. Biochem. Biotechnol. 184:987-1008. https://doi.org/10.1007/ s12010-017-2600-7.

Melendez, J., M. Grogg, and Y. Zheng. 2011. Signaling role of CDC42 in regulating mammalian physiology. J. Biol. Chem. 286:23752381. https://doi.org/10.1074/jbc.R110.200329.

Mezera, M. A., C. S. Hamm, C. A. Gamarra, R. S. Gennari, A. B. Prata, R. Sartori, and M. C. Wiltbank. 2019. Profiles of prostaglandin F2 $\alpha$ metabolite (PGFM) in dairy cattle during luteal regression and pregnancy: implications for corpus luteum maintenance. Biol. Reprod. 101:76-90. https://doi.org/10.1093/biolre/ioz074.

Mondal, M., B. Schilling, J. Folger, J. P. Steibel, H. Buchnick, Y. Zalman, J. J. Ireland, R. Meidan, and G. W. Smith. 2011. Deciphering the luteal transcriptome: potential mechanisms mediating stage-specific luteolytic response of the corpus luteum to prostaglandin F-2 alpha. Physiol. Genomics 43:447-456. https://doi.org/ 10.1152/physiolgenomics.00155.2010.

Mossberg, K., P. A. Svensson, O. Gidlof, D. Erlinge, S. Jern, and H. Brogren. 2016. Normalization of qPCR in platelets - YWHAE a potential generic reference gene. Platelets 27:729-734. https://doi .org/10.1080/09537104.2016.1180349.

Nygard, A. B., C. B. Jorgensen, S. Cirera, and M. Fredholm. 2007. Selection of reference genes for gene expression studies in pig tissues using SYBR green qPCR. BMC Mol. Biol. 8:67. https://doi.org/ 10.1186/1471-2199-8-67.

Ochoa, J. C., F. Peñagaricano, G. M. Baez, L. F. Melo, J. C. Motta, A. G. Guerra, R. Meidan, J. C. P. Ferreira, R. Sartori, and M. C. Wiltbank. 2018. Mechanisms for rescue of corpus luteum dur- ing pregnancy: Gene expression in bovine corpus luteum following intrauterine pulses of prostaglandins E1 and F2 $\alpha$. Biol. Reprod. 98:465-479. https://doi.org/10.1093/biolre/iox183.

Pfaffl, M. W., A. Tichopad, C. Prgomet, and T. P. Neuvians. 2004. Determination of stable housekeeping genes, differentially regulated target genes and sample integrity: BestKeeper - Excel-based tool using pair-wise correlations. Biotechnol. Lett. 26:509-515. https://doi.org/10.1023/B:BILE.0000019559.84305.47.

Pfeffer, S., J. Dudek, M. Schaffer, B. G. Ng, S. Albert, J. M. Plitzko, W. Baumeister, R. Zimmermann, H. H. Freeze, B. D. Engel, and F. Forster. 2017. Dissecting the molecular organization of the translocon-associated protein complex. Nat. Commun. 8:14516. https://doi.org/10.1038/ncomms14516.

Pols, M. S., and J. Klumperman. 2009. Trafficking and function of the tetraspanin CD63. Exp. Cell Res. 315:1584-1592. https://doi.org/ 10.1016/j.yexcr.2008.09.020.

Popovici, V., D. R. Goldstein, J. Antonov, R. Jaggi, M. Delorenzi, and P. Wirapati. 2009. Selecting control genes for RT-QPCR using public microarray data. BMC Bioinformatics 10:42. https://doi .org/10.1186/1471-2105-10-42.

Pyott, S. M., U. Schwarze, H. E. Christiansen, M. G. Pepin, D. F. Leistritz, R. Dineen, C. Harris, B. K. Burton, B. Angle, K. Kim, M. D. Sussman, M. Weis, D. R. Eyre, D. W. Russell, K. J. McCarthy, R. D. Steiner, and P. H. Byers. 2011. Mutations in PPIB (cyclophilin B) delay type I procollagen chain association and result in perinatal lethal to moderate osteogenesis imperfecta phenotypes. Hum. Mol. Genet. 20:1595-1609. https://doi.org/10.1093/ hmg/ddr037.

Rekawiecki, R., J. Rutkowska, and J. Kotwica. 2012. Identification of optimal housekeeping genes for examination of gene expression in bovine corpus luteum. Reprod. Biol. 12:362-367. https://doi.org/ 10.1016/j.repbio.2012.10.010.

Risso, D., J. Ngai, T. P. Speed, and S. Dudoit. 2014. Normalization of RNA-seq data using factor analysis of control genes or samples. Nat. Biotechnol. 32:896-902. https://doi.org/10.1038/nbt.2931.

Rufini, A., M. V. Niklison-Chirou, S. Inoue, R. Tomasini, I. S. Harris, A. Marino, M. Federici, D. Dinsdale, R. A. Knight, G. Melino, and T. W. Mak. 2012. TAp73 depletion accelerates aging through metabolic dysregulation. Genes Dev. 26:2009-2014. https://doi .org/10.1101/gad.197640.112.

Sakumoto, R., K. G. Hayashi, M. Hosoe, K. Iga, K. Kizaki, and K. Okuda. 2015. Gene expression profiles in the bovine corpus luteum (CL) during the estrous cycle and pregnancy: Possible roles of chemokines in regulating CL function during pregnancy. J. Reprod. Dev. 61:42-48. https://doi.org/10.1262/jrd.2014-101.

Samiullah, S., J. Roberts, and S. B. Wu. 2017. Reference gene selection for the shell gland of laying hens in response to time-points of eggshell formation and nicarbazin. PLoS One 12:e0180432. https:/ /doi.org/10.1371/journal.pone.0180432.

Schoen, K., J. Plendl, C. Gabler, and S. Kaessmeyer. 2015. Identification of stably expressed reference genes for RT-qPCR data normalization in defined localizations of cyclic bovine ovaries. Anat Histol. Embryol. 44:200-211. https://doi.org/10.1111/ahe.12128.

Sesen, J., J. Casaos, S. J. Scotland, C. Seva, T. S. K. Eisinger-Mathason, and N. Skuli. 2017. The bad, the good and eIF3e/INT6. Front. Biosci. 22:4469. https://doi.org/10.2741/4469.

Shaydurov, V. A., A. Kasianov, and A. P. Bolshakov. 2018. Analysis of housekeeping genes for accurate normalization of qPCR data during early postnatal brain development. J. Mol. Neurosci. 64:431439. https://doi.org/10.1007/s12031-018-1037-y.

Song, G., T. E. Spencer, and F. W. Bazer. 2006. Progesterone and interferon- $\tau$ regulate cystatin $\mathrm{C}$ in the endometrium. Endocrinology 147:3478-3483. https://doi.org/10.1210/en.2006-0122.

Steele, B. K., C. Meyers, and M. A. Ozbun. 2002. Variable expression of some "housekeeping" genes during human keratinocyte differentiation. Anal. Biochem. 307:341-347. https://doi.org/10.1016/ S0003-2697(02)00045-3.

Suzuki, T., P. Higgins, and D. Crawford. 2000. Control selection for RNA quantitation. Biotechniques 29:332-337. https://doi.org/10 $.2144 / 00292$ rv02. 
Too, I. H. K., and M. H. T. Ling. 2011. Signal peptidase complex subunit 1 and hydroxyacyl-CoA dehydrogenase beta subunit are suitable reference genes in human lungs. ISRN Bioinform. 2012:790452. https://doi.org/10.5402/2012/790452.

Trapnell, C., A. Roberts, L. Goff, G. Pertea, D. Kim, D. R. Kelley, H. Pimentel, S. L. Salzberg, J. L. Rinn, and L. Pachter. 2012 Differential gene and transcript expression analysis of RNA-seq experiments with TopHat and Cufflinks. Nat. Protoc. 7:562-578. https://doi.org/10.1038/nprot.2012.016.

Tsai, S. J., K. Kot, O. J. Ginther, and M. C. Wiltbank. 2001. Temporal gene expression in bovine corpora lutea after treatment with PGF2 alpha based on serial biopsies in vivo. Reproduction 121:905-913. https://doi.org/10.1530/rep.0.1210905.

van den Driesche, S., M. Myers, E. Gay, K. J. Thong, and W. C. Duncan. 2008. HCG up-regulates hypoxia inducible factor-1 alpha in luteinized granulosa cells: Implications for the hormonal regulation of vascular endothelial growth factor A in the human corpus luteum. Mol. Hum. Reprod. 14:455-464. https://doi.org/10.1093/ molehr/gan040.

van Hemert, M. J., H. Y. de Steensma, and G. P. H. van Heusden. 2001. 14-3-3 proteins: Key regulators of cell division, signalling and apoptosis. BioEssays 23:936-946. https://doi.org/10.1002/ bies. 1134

Vandesompele, J., K. De Preter, F. Pattyn, B. Poppe, N. Van Roy, A. De Paepe, and F. Speleman. 2002. Accurate normalization of real-time quantitative RT-PCR data by geometric averaging of multiple internal control genes. Genome Biol. 3:research0034.1.
Wang, Z., M. Gerstein, and M. Snyder. 2009. RNA-Seq: A revolutionary tool for transcriptomics. Nat. Rev. Genet. 10:57-63. https:// doi.org/10.1038/nrg2484.

Wenger, R. H., A. Rolfs, P. Spielmann, D. R. Zimmermann, and M. Gassmann. 1998. Mouse hypoxia-inducible factor-1 alpha is encoded by two different mRNA isoforms: Expression from a tissuespecific and a housekeeping-type promoter. Blood 91:3471-3480. https://doi.org/10.1182/blood.V91.9.3471.

Xu, H., C. Li, Q. Zeng, I. Agrawal, X. Zhu, and Z. Gong. 2016. Genome-wide identification of suitable zebrafish Danio rerio reference genes for normalization of gene expression data by RT-qPCR. J. Fish Biol. 88:2095-2110. https://doi.org/10.1111/jfb.12915.

Ye, J., G. Coulouris, I. Zaretskaya, I. Cutcutache, S. Rozen, and T. L. Madden. 2012. Primer-BLAST: A tool to design target-specific primers for polymerase chain reaction. BMC Bioinformatics 13:134. https://doi.org/10.1186/1471-2105-13-134.

\section{ORCIDS}

M. A. Mezera ๑ https://orcid.org/0000-0002-1037-9902

A. D. Beard ( https://orcid.org/0000-0002-2590-7296

M. C. Wiltbank (ํ) https://orcid.org/0000-0001-8188-0991 